\title{
STRIA TERMINALIS LESIONS ALTER THE TEMPORAL PATTERN OF COPULATORY BEHAVIOR IN THE MALE GOLDEN HAMSTER
}

\author{
MICHAEL N. LEHMAN, J. BRADLEY POWERS and SARAH S. WINANS
}

Department of Anatomy and Cell Biology, University of Michigan, Ann Arbor, MI 48109 and (J.P.B.) Department of Psychology, Vanderbilt University, Nashville, TN (U.S.A.)

(Received July 1st, 1982)

(Revised version received November, 1982)

(Accepted November 26th, 1982)

Key words: stria terminalis - amygdala - vomeronasal system - olfactory system - male sexual behavior - hamster

SUMMARY

The mating behavior of a group of male golden hamsters was observed before and after bilateral electrolytic lesions or knife cuts interrupting the stria terminalis (ST). Whereas males with bilateral lesions of the medial nucleus of the amygdala had previously been observed to stop mating, a majority of hamsters with bilateral ST destruction, whether by electrolytic lesions or knife cuts, continued to display mounts, intromissions, and ejaculations during tests over a two month postoperative period. ST-lesioned males did, however, display a distinctly altered pattern of copulation over the course of postoperative testing, consisting of an increase in mount latency during the first week, an increase in the inter-intromission interval during the second week, and an increase in the number of intromissions preceding ejaculation during the third and subsequent weekly tests. Males with bilateral lesions of the caudal amygdala, which damaged the amygdaloid exit of the ST, displayed alterations in copulatory behavior similar to those seen after bilateral ST destruction at a more rostral level.

\section{INTRODUCTION}

The stria terminalis (ST) has long been considered the major efferent pathway by which the amygdala modulates activity in the preoptic area and medial hypothalamus, brain regions critical for a wide variety of motivated behaviors [ 1 , 
$12,22]$. In this regard the ST has often been implicated in the influence of the corticomedial amygdala on sexual behavior and those neuroendocrine events associated with reproduction [7, 11, 30, 31]. A major source of amygdaloid efferents in the ST is the medial nucleus $[5,14,16]$, and recent experiments in our laboratory have demonstrated that, in the male hamster, this nucleus plays an important role in controlling male sexual behavior [18, 19]. Bilateral electrolytic destruction of the medial nucleus in these animals eliminates all components of copulatory behavior for at least 3 months postoperatively, and severely reduces the male's anogenital investigation of the receptive female during those encounters $[18,19]$.

Further, the medial nucleus has both afferent and efferent connections with areas of the brain which are also essential for mating behavior in the male hamster. Major sources of input to the medial nucleus are from the vomeronasal system, via the accessory olfactory bulb [4, 28], and from the main olfactory system, via secondary and tertiary nuclei in the olfactory pathway $[15,16]$. Destruction of both of these systems, either by bilateral olfactory bulbectomy [25], or bilateral peripheral deafferentation [32], eliminates copulation in the male hamster. Efferents of the medial nucleus via the ST project to the bed nucleus of the ST (BNST) and the medial preoptic-anterior hypothalamic junction (MPOAH) [14], two brain areas which have also been shown to control male mating behavior [ 17 . $22]$ and which, like the medial nucleus $[6,29]$, are major targets for the actions of androgens [27].

In light of these anatomical connections and functional similarities, we have suggested that, in the hamster, chemosensory information processed in the medial nucleus exerts its influence over mating behavior by modulating activity in the BNST and/or MPOAH via the ST. We therefore hypothesized that bilateral destruction of the ST would eliminate male sexual behavior in the hamster. To test this hypothesis, we examined the mating behavior of male hamsters before and after either bilateral electrolytic lesions of the ST, or bilateral knife cuts which transected the ST. Because neuroanatomical tract tracing studies, using both autoradiographic and silver degeneration techniques, have demonstrated that efferents of the medial nucleus travel as a topographically organized fascicle in the ventromedial portion of the ST $[5,16]$, we analyzed separately the behavior of animals with bilateral lesions which only damaged the medial half of the ST and animals with bilateral lesions that destroyed the entire ST. In addition, because those medial nucleus lesions which eliminated mating behavior in previous studies $[18,19]$ were rostrally-situated and did not entirely destroy the amygdaloid exit of the ST, we also examined the mating behavior of male hamsters with bilateral lesions placed in the caudal corticomedial amygdala (CMA) which interrupted the amygdaloid exit of the ST, and compared the postoperative behavior of these animals to those with more rostral bilateral lesions of the ST. 


\section{Animals}

Adult male hamsters weighing approximately $120 \mathrm{~g}$ were obtained from Engle Laboratory Animals (Farmersburg, IN). Animals were housed individually with food and water available ad libitum and exposed to a $14 \mathrm{~h}$ light : $10 \mathrm{~h}$ dark illumination cycle.

\section{Mating behavior tests}

Tests were conducted 2-3 h into the dark portion of the animal's illumination cycle. Mating behavior was observed after introducing a behaviorally receptive female into a clean plexiglass cage containing the experimental male hamster. Adult ovariectomized female hamsters were made sexually receptive by sequential subcutaneous injections of estradiol benzoate $(10 \mu \mathrm{g})$ and progesterone $(200 \mu \mathrm{g})$ dissolved in $0.1 \mathrm{ml}$ sesame oil and administered 42 and $6 \mathrm{~h}$, respectively, before behavior testing. The female's receptivity was confirmed prior to mating tests by exposure to a stud male.

The latency and frequency of all mounts, intromissions, and ejaculations displayed by the male hamster during the mating test were recorded. Additionally, for some animals, the duration of the male's sniffing and licking investigation of the female's head, flank, and anogenital region was measured. Latency data were recorded using the timer function of a Hewlett-Packard HP-55 calculator; frequency data were recorded with a series of mounted counters; duration data were recorded with a series of contact-activated electromechanical timers and counters. Observations continued for $10 \mathrm{~min}$, or until the male had achieved two ejaculations. Post-ejaculatory intervals were calculated by subtracting the latency of the male's first ejaculation from his latency to initiate mounting following the refractory period. Mean inter-intromission intervals were calculated by subtracting the male's intromission latency from his ejaculation latency for a given series, and dividing by the number of intromissions prior to ejaculation.

Experimental males were screened for normal mating behavior and tested as described above twice over a period of 1 week prior to surgery. An initial experimental group was tested weekly for 2 weeks following surgery; in a replication, a second group was tested for 3 weeks. Several experimental animals were given additional weekly mating tests for up to 2 months following surgery.

\section{Brain lesions and knife cuts}

Electrolytic lesions of the stria terminalis (ST) were produced in 32 anesthetized hamsters (Nembutal, $75 \mathrm{mg} / \mathrm{kg}$, i.p.) by stereotaxically lowering an insect pin electrode (tip diameter $0.37 \mathrm{~mm}$ ), insulated except for $0.5 \mathrm{~mm}$ at the tip, and delivering an anodal DC current of $2 \mathrm{~mA}$ for $15 \mathrm{sec}$. Stereotaxic coordinates for ST lesions with bregma and lambda in a horizontal plane were AP $+0.2, \mathrm{ML}$ 
\pm 2.0 from bregma, and DV -5.1 to -5.5 from dura. Sham ST lesions in 8 hamsters were accomplished by lowering the electrode to $1 \mathrm{~mm}$ above experimental coordinates without the delivery of current. In 6 animals transection of the ST was accomplished by lowering a stainless steel razor blade with its cutting edge (1.2 $\mathrm{mm}$ in length) positioned in a coronal plane. Stereotaxic coordinates were the same used for electrolytic lesions. Control knife cuts were made in two hamsters by lowering the razor blade to $2 \mathrm{~mm}$ above experimental coordinates.

Electrolytic lesions of the caudal corticomedial amygdala (CMA) were produced in 6 hamsters using the same insect pin electrodes described above, delivering an anodal DC current of $1.5 \mathrm{~mA}$ for $15 \mathrm{sec}$. Stereotaxic coordinates for caudal CMA lesions with bregma and lambda in a horizontal plane were AP - 0.6 to $-1.0, \mathrm{ML} \pm 2.9$ from bregma, and DV -7.8 from dura. Sham CMA lesions in 8 hamsters were accomplished in the same manner as described for sham ST lesions.

\section{Histological procedures}

At the end of postoperative testing, 20 of those animals with bilateral ST lesions or knife cuts were used for a related experiment in which horseradish peroxidase (HRP) was applied into the bed nucleus of the stria terminalis or medial preoptic-anterior hypothalamic junction [20]. These animals survived $48 \mathrm{~h}$ following surgery to introduce the HRP and then were perfused according to procedures appropriate for HRP histochemistry using the tetramethylbenzidine method. All other animals were perfused with a wash of $0.9 \%$ physiological saline, followed by $10 \%$ phosphate buffered formalin. All brains were cut coronally in $50 \mu \mathrm{m}$ sections on a freezing microtome, and sections through the area of the lesion at $100 \mu \mathrm{m}$ intervals were mounted on gel-coated slides, dried, and stained with cresyl violet.

Using a microprojector, each lesion in every animal was reconstructed by tracings from sequential sections. Caudal CMA lesions were further analyzed by plotting each lesion on a set of 18 standard coronal sections through the amygdala. Using a computerized image analysis system (Quantimet 720 interfaced with a DEC PDP-11 computer), we then measured the damaged area and the overall area of each amygdaloid nucleus for each standard section. The percentage of bilateral damage to a specific nucleus was calculated by dividing the lesioned area of the nucleus bilaterally, as summated from sequential standard sections, by the total area of that nucleus bilaterally summated from the same standard sections.

\section{RESULTS}

Stria terminalis lesion and knife cut histology

Male hamsters with electrolytic lesions of the stria terminalis (ST) were divided into four groups based on the histological analysis of the placement and 
extent of their lesions: (a) animals with bilateral sham ST lesions (Fig. 1a); (b) animals with bilateral lesions which destroyed the entire ST, and damaged the adjacent fimbria, and reticular nucleus of the thalamus (Fig. 1b); (c) animals with bilateral lesions which destroyed the medial half of the ST, and damaged the fimbria (Fig. 1c); and (d) animals with misplaced lesions which damaged the anteroventral and anterodorsal nuclei of the thalamus, as well as part of the fimbria, but left the ST intact (Fig. 1d). It should be noted of the latter group that although the ST appeared intact in these animals, some medial fibers of the ST may have been damaged since in most cases gliotic tissue directly abutted this area (see Fig. 1d). Hence these animals were referred to as a group with 'minimal ST damage'.

Razor blade knife cuts of the ST, in all cases, completely transected the entire ST, as well as part of the fimbria, corpus callosum, and overlying neocortex. Behavioral results showed that male hamsters with ST knife cuts displayed a pattern of mating deficits identical to those animals with complete ST destruction produced by electrolytic lesions (group b in Fig. 1), thus these two groups of males were combined for statistical analysis. Control knife cuts lowered to $2 \mathrm{~mm}$ above experimental coordinates transected parts of the fimbria, corpus callosum, and neocortex. Consequently, animals bearing these knife cuts provided a control for damage to the fimbria, both in animals with complete knife cuts of the ST, and in animals with electrolytic ST lesions.

\section{Copulatory behavior following stria terminalis lesions and knife cuts}

Approximately $75 \%$ of male hamsters with complete bilateral destruction of the ST continued to display all the components of normal copulatory behavior (mounts, intromissions, and ejaculations) during postoperative tests (Fig. 2B), although these animals did show temporal alterations in copulation that will be examined in detail below. In contrast to males with complete ST destruction, only $25 \%$ of male hamsters with bilateral lesions which destroyed the medial portion of the ST continued to mate during their first and second postoperative test (Fig. 2C). However, 3 of the 4 animals in this group that were given a third postoperative test displayed all the components of normal copulatory behavior.

A majority of male hamsters with bilateral lesions which caused minimal damage to the ST continued to display all the components of copulatory behavior during all three postoperative tests. Furthermore, the percentage of animals in this group that mated on a given test was comparable to that of males with complete ST lesions (Fig. 2D). All males with sham lesions or control knife cuts continued to mate during all postoperative tests, with the exception of one animal with bilateral sham lesions during the second postoperative test (Fig. 2A). In summary then, males with either complete bilateral destruction of the ST or minimal ST damage, for the most part, continued to display all the components of normal 


\section{Sham control}

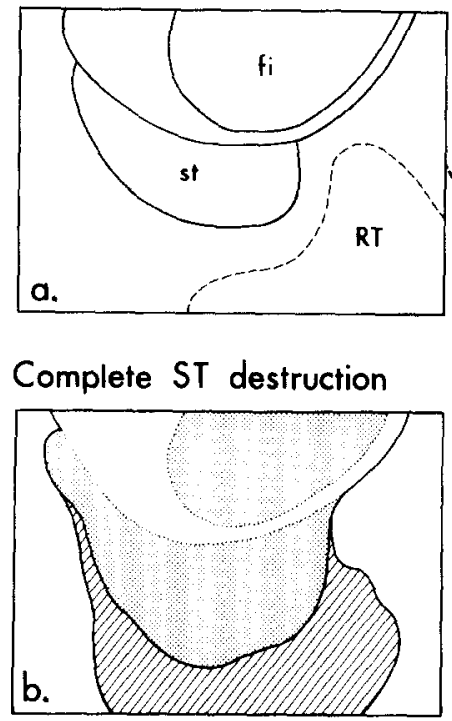

\section{Medial ST damage}

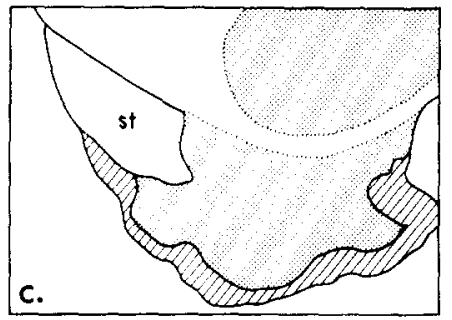

\section{Minimal ST damage}
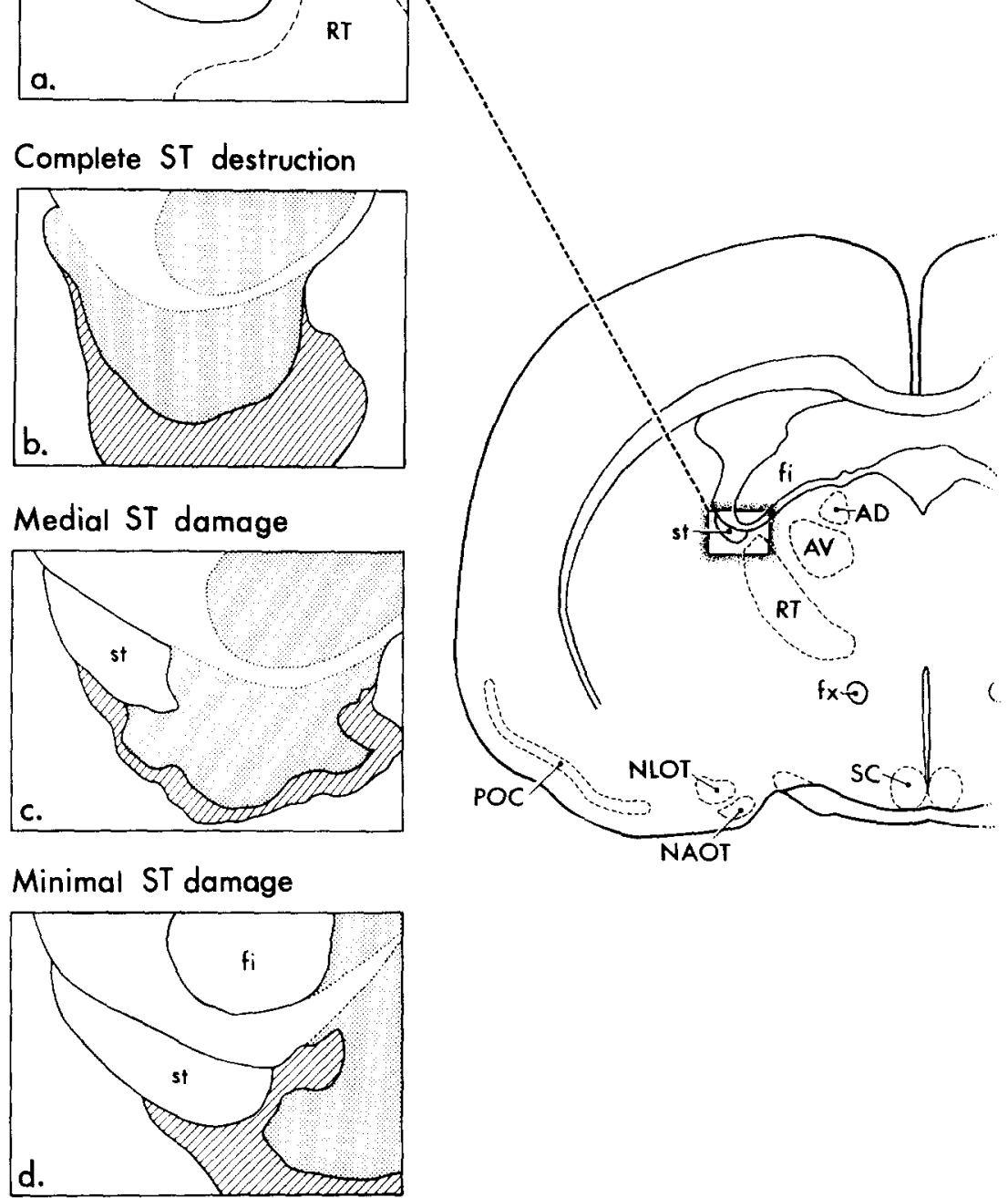

Fig. 1. Tracings of coronal brain sections from 4 representative hamsters illustrating (a) the normal location of the stria terminalis (st) and adjacent structures, and the placement and extent of lesions which destroyed (b) the entire stria terminalis; (c) the medial half of the stria terminalis, or (d) caused minimal strial damage. Stippled area, necrotic or absent tissue at the center of the lesion; hatched area, gliotic tissue. Abbreviations: AD, anterodorsal nucleus of the thalamus; AV, anteroventral nucleus of the thalamus; fi, fimbria; fx, fornix; NAOT, nucleus of the accessory olfactory tract; NLOT, nucleus of the lateral olfactory tract; POC, primary olfactory cortex; RT, reticular nucleus of the thalamus; SC, suprachiasmatic nucleus of the hypothalamus. 


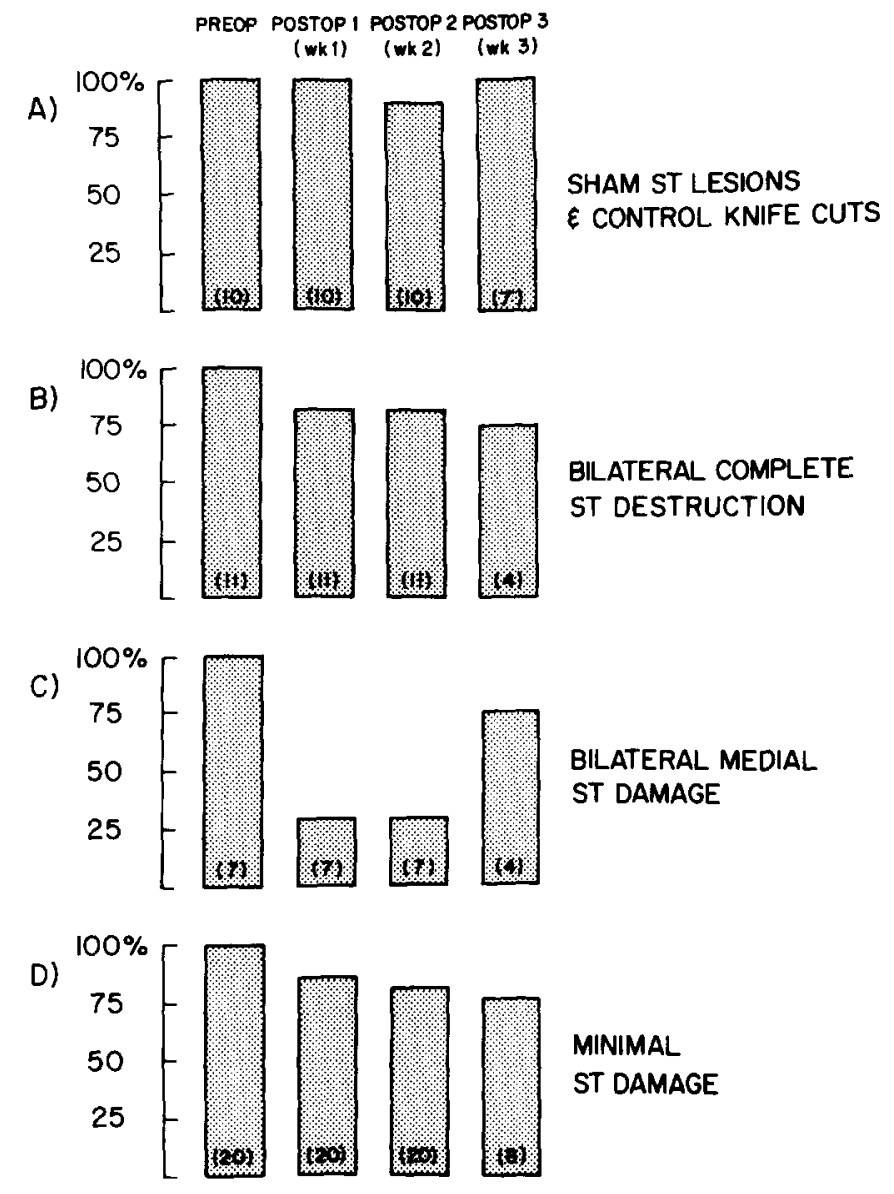

Fig. 2. Percentage of male hamsters displaying mounts, intromissions, and ejaculations during mating tests, before and after sham lesions (A) or bilateral lesions of the stria terminalis (B-D) which correspond respectively to those illustrated in Fig. 1 (b-d). Numbers in parentheses at the bottom of each histogram indicate sample size for each test. Preoperative values in this figure, Tables I and II, and Figs. 4 and 5 , are the results of the second preoperative test.

copulation during postoperative tests. Only in the group of males with bilateral damage to the medial half of the ST did a majority of animals show an absence of copulatory behavior during any given postoperative test; even so, in this experimental group, a majority of males did display copulatory behavior during at least one of three postoperative tests. Additionally, it should be noted that those males of this group that continued to mate were not the same individuals on each postoperative test; $70 \%$ of all male hamsters with medial ST lesions mated during at least one of three postoperative tests. This reduction in behavior is similar to that seen in some males after bilateral vomeronasal nerve cuts [32] but is not as severe as the total loss of behavior following bilateral lesions of the medial amygdaloid nucleus [18, 19]. 


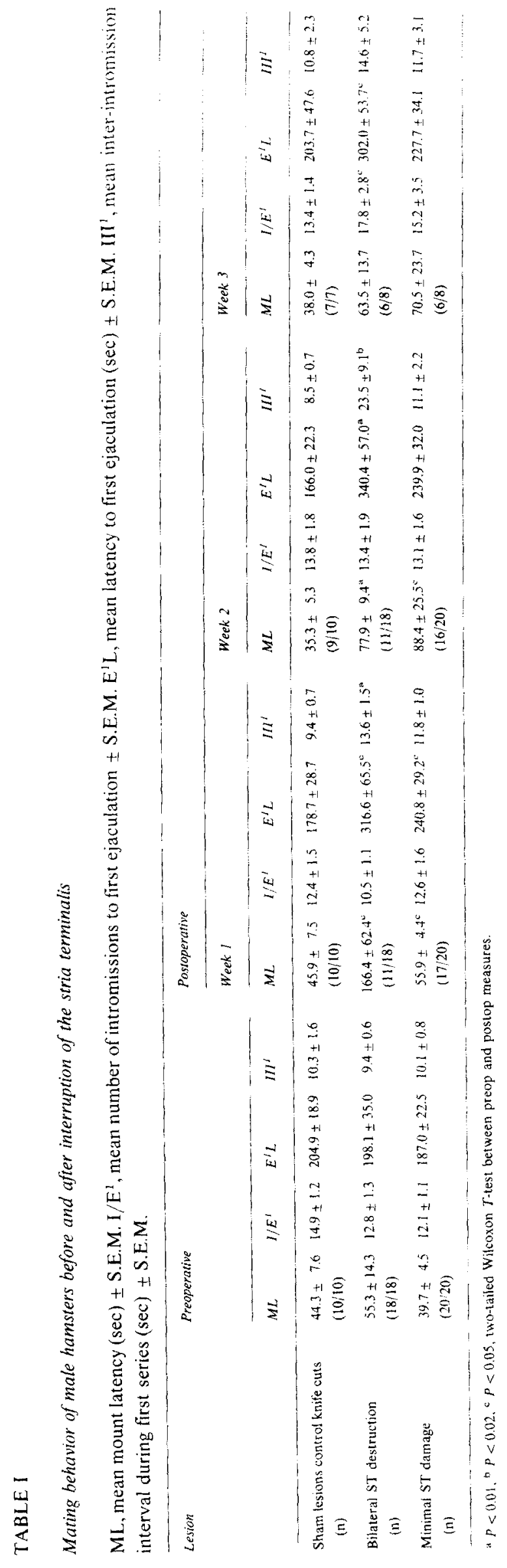




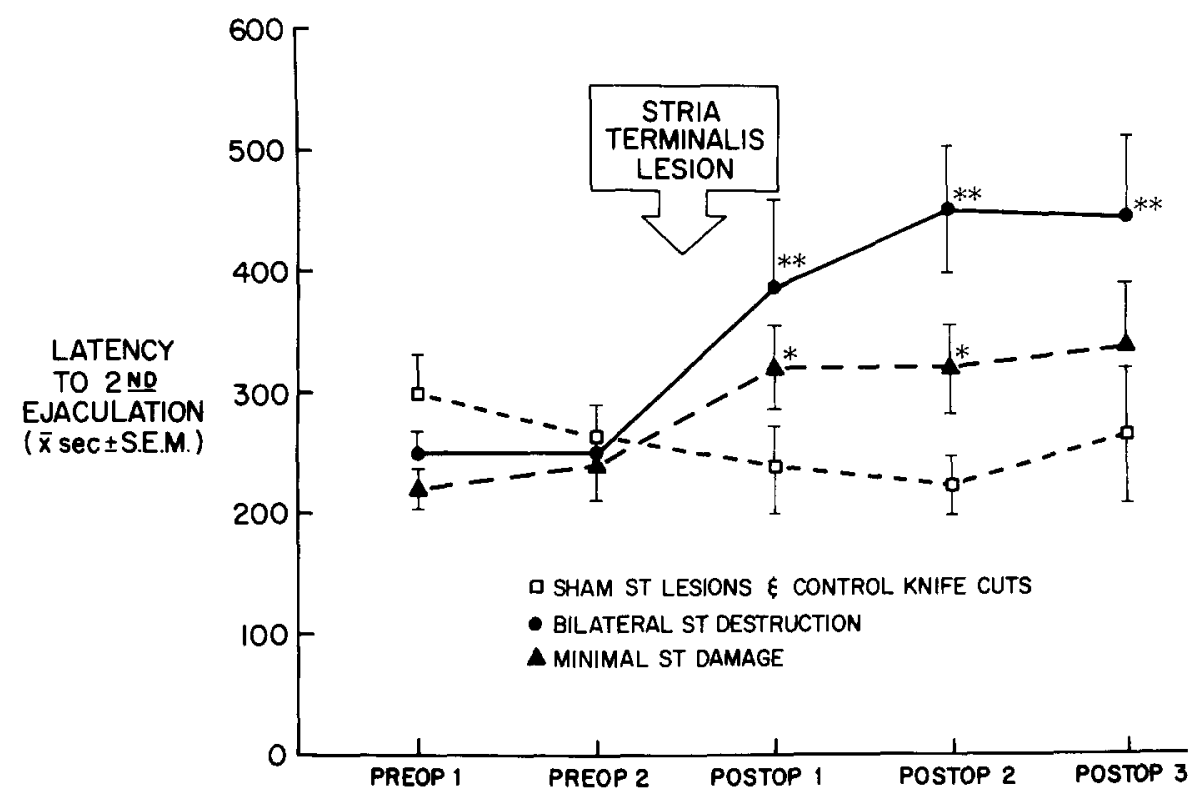

Fig. 3. Mean latency to second ejaculation during mating tests before and after stria terminalis lesions or sham lesions. See Table I for ' $n$ ' values. ${ }^{* *} P<0.01,{ }^{*} P<0.05$, two-tailed Wilcoxon $T$-test between 'PREOP 2' measures and postop measures.

Moreover, those males with medial ST damage that continued to mate during postoperative tests showed the same pattern of temporal changes in copulatory behavior as males with complete bilateral destruction of the ST. Since the individuals in these two groups of males were statistically indistinguishable, in all tests in which mating occurred, on all measures of copulatory behavior in which they differed from sham controls $(P<0.01$, Mann-Whitney U-test $)$, they were combined for analysis. Collectively they are hereafter referred to as males with 'bilateral ST destruction' (Table I). Notably, all of these males showed a significant increase in their latency to first ejaculation $\left(E^{1} L\right)$ (Table I) and second ejaculation ( $\left.E^{2} L\right)$ (Fig. 3) throughout all postoperative tests. In addition, during some but not all postoperative tests, they showed significant increases in their latency to initiate mounting of the female (ML), in the length of their mean inter-intromission interval during the first series $\left(\mathrm{III}^{1}\right)$, and in the number of intromissions preceding their first ejaculation $\left(\mathrm{I} / \mathrm{E}^{1}\right)$ (Table $\mathrm{I}$ ). Although not shown in Table I, males with bilateral ST destruction also displayed a significant increase in the length of their post-ejaculatory interval (PEI) following the first ejaculation, from a mean of $33 \mathrm{sec}$ preoperatively to $42 \mathrm{sec}$ during the second and third postoperative tests $(P<0.01$, Wilcoxon $T$-test $)$.

Male hamsters with minimal ST damage displayed similar but smaller increases in the same measures of copulatory behavior as those males with bilateral ST destruction (Table I). For example, these males also showed an 
increase in $\mathrm{E}^{2} \mathrm{~L}$ during all postoperative tests (Fig. 3), however, in this group, the increase compared to preoperative levels was only statistically significant during the first and second, but not the third postoperative test. Similarly, males with minimal ST damage displayed postoperative increases in $\mathrm{ML}, \mathrm{I} / \mathrm{E}^{1}$, and $\mathrm{III}^{1}$ that mirrored those seen in animals with bilateral ST destruction, but in most cases these changes did not reach comparable levels of statistical significance (Table I). Animals with sham lesions or control knife cuts displayed none of the alterations in the temporal pattern of copulation characteristic of animals with either bilateral ST destruction or minimal ST damage (Table I). Because the control knife cuts damaged the fimbria, interruption of these fibers was probably not the major cause of copulatory deficits seen in the experimental males.

After analyzing these changes in the temporal pattern of copulation, it was apparent that the increase in latency to first and second ejaculation seen in animals with bilateral ST destruction and minimal ST damage, could be attributed to specific alterations in one or more component of copulatory behavior over the course of postoperative testing. Those factors which contributed to an increase in $E^{1} \mathrm{~L}$ during each of the three postoperative tests can be understood through Fig. 4 , in which chronograms created from the data of Table I illustrate the mean timing of copulatory behaviors leading to the first ejaculation in animals with bilateral ST destruction. From the data represented in this figure and in Fig. 5A, it is clear that the major factor responsible for the increase in $\mathrm{E}^{1} \mathrm{~L}$ during the first postoperative test was a significant increase in the mounting latency of these males $(P<0.01$, Wilcoxon $T$-test). During the second postoperative test, mount latency

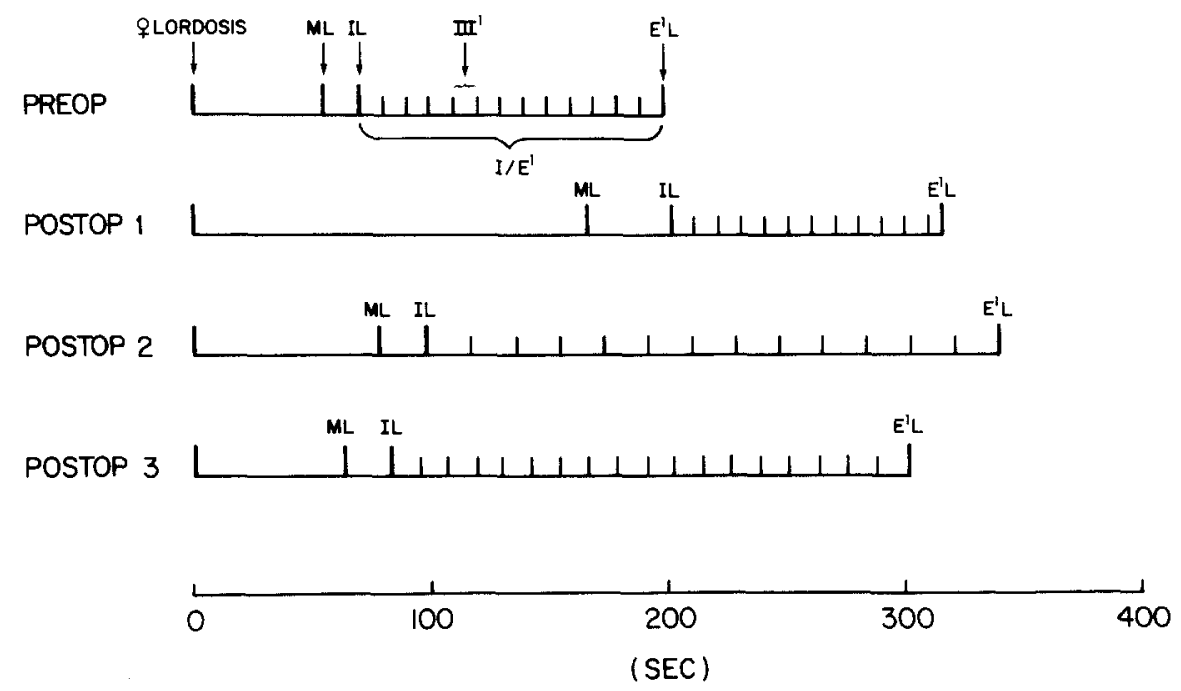

Fig. 4. Chronograms illustrating the mean timing of copulatory behaviors leading to the first ejaculation before and after bilateral destruction of the stria terminalis. IL, mean latency to first intromission; all other abbreviations the same as in Table I. 
was reduced (Fig. 5A) and a significant increase in the mean inter-intromission interval of these animals accounted for most of the increase in $E^{1} L$ (Fig. 4; Fig. 5B). Finally, during the third postoperative test both mount latency and the mean inter-intromission interval were reduced (Figs. 5A, B) and the increase in $E^{1} \mathrm{~L}$ could be attributed to a significant increase in the mean number of
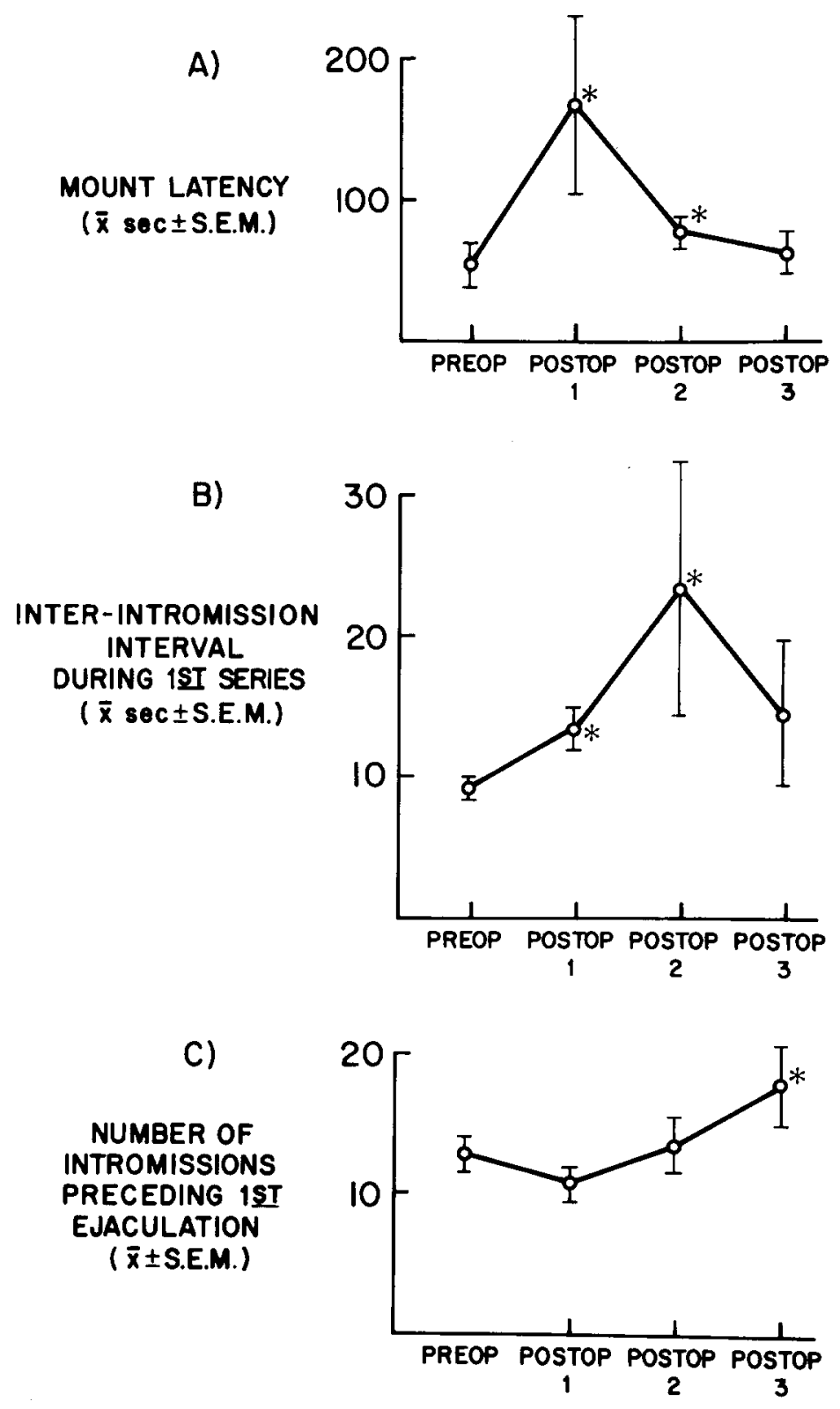

Fig. 5. Mean mount latency (A), mean inter-intromission interval (B), and mean number of intromissions preceding first ejaculation $(C)$ seen before and after bilateral destruction of the stria terminalis. See Table I for ' $n$ ' values. ${ }^{*} P<0.05$, two-tailed Wilcoxon $T$-test between preop and postop measures. 
intromissions preceding the first ejaculation (Fig. 4; Fig. 5C). For those males with bilateral ST destruction which received mating tests over an extended period (up to 2 months) following the third postoperative test, an increase in the number of intromissions preceding the first ejaculation continued to be the primary factor contributing to a lengthening of the $\mathrm{E}^{1} \mathrm{~L}$. It should also be noted that the significant increase in latency to second ejaculation displayed by animals with bilateral ST destruction (Fig. 3) can be attributed both to those alterations described above and to the significant increase, described previously, in the length of the PEI displayed by these animals during the second and third postoperative tests.

\section{Copulatory behavior following caudal corticomedial amygdaloid lesions}

Histological analysis of the placement and extent of lesions in the caudal corticomedial amygdala (CMA) revealed that these lesions interrupted the amygdaloid exit of the ST, and bilaterally damaged the caudal part of the medial nucleus of the amygdala (average $=45 \%$; range $=34-82 \%$ ), the posteromedial cortical nucleus of the amygdala (average $=39 \%$; range $=15-52 \%$ ), the amygdalo-hippocampal area (average $=50 \%$; range $=12-70 \%$ ), and, in one animal, $6 \%$ of the central nucleus of the amygdala (see Fig. 1B in ref. 21 for representative lesion).

Male hamsters with bilateral caudal CMA lesions displayed postoperative mating deficits that were in several respects similar to those displayed by males with bilateral lesions that damaged the medial half of the ST. Like those animals with medial ST lesions (see Fig. 2B), approximately half of those males with caudal CMA lesions stopped mating on a given postoperative test (note ' $n$ ' values in Table II). Once again, those animals that did mate were not the same individuals on each test; in fact, every male with bilateral caudal CMA lesions displayed a complete sequence of copulatory behaviors during at least one of three postoperative tests.

Furthermore, those males with caudal CMA lesions that did continue to mate during postoperative tests showed temporal alterations in their pattern of copulation that paralleled those postoperative alterations displayed by males with either medial ST lesions or complete ST lesions (compare 'bilateral ST destruction' group of Table I with 'caudal CMA lesion' group of Table II). Males with caudal CMA lesions, like males with bilateral ST destruction, displayed increases in mount latency, latency to first and second ejaculation, the length of their mean inter-intromission interval during the first series, and the number of intromissions preceding the first ejaculation. Because of the small number of males with caudal CMA lesions, many of these increases approached but did not reach levels of statistical significance (Table II). Nevertheless, these postoperative alterations in one or more component of copulatory behavior occurred in approximately the same sequence over the course of testing as they did for animals with bilateral ST 


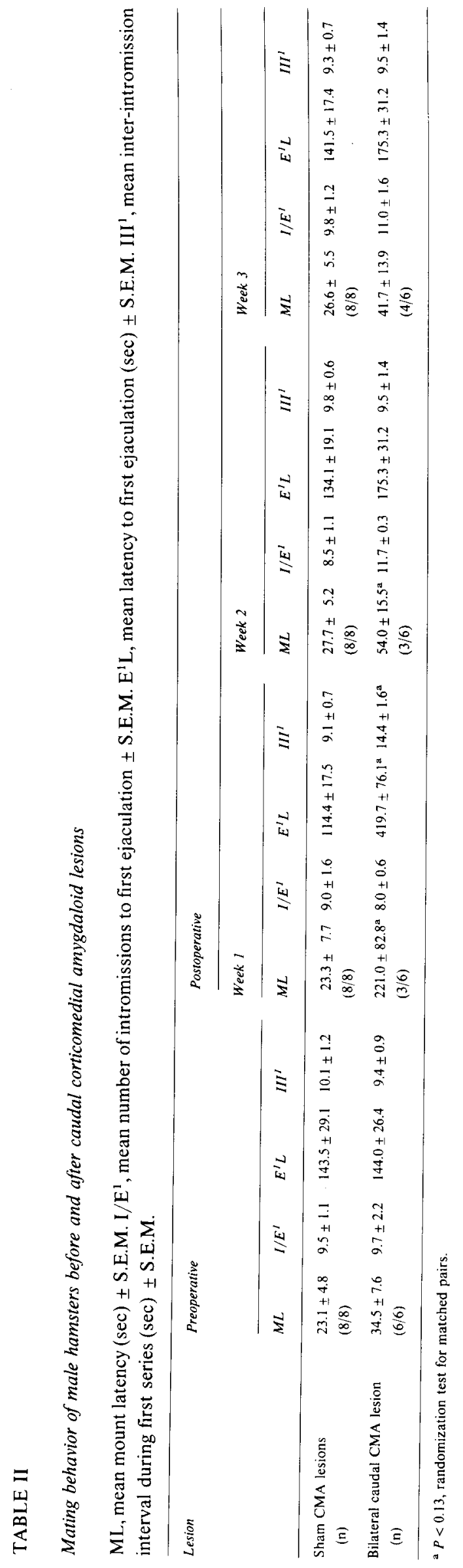


destruction, and contributed to a lengthening of ejaculation latencies in a similar manner to that previously described. Males with sham CMA lesions continued to mate normally in postoperative tests (Table II), and displayed none of the alterations in the pattern of copulation that characterized males with caudal CMA lesions or bilateral ST destruction.

Anogenital investigation following stria terminalis lesions or caudal corticomedial amygdaloid lesions

We measured the male's anogenital investigation of the female during mating tests in all males with caudal CMA lesions or sham CMA lesions, and also in a subset of male hamsters with either bilateral ST destruction or minimal ST damage. Because the duration of postoperative mating tests varied for males with ST or caudal CMA lesions, we measured investigatory behavior as the time spent sniffing and licking each region of the female's body per minute of test. Male hamsters with sham lesions showed a decrease in their mean rate of anogenital investigation, averaged across all postoperative tests, of $25 \%$ compared to preoperative levels (Fig. 6). This decrease is probably due to the experience of repeated exposure to a receptive female over the course of pre- and postoperative

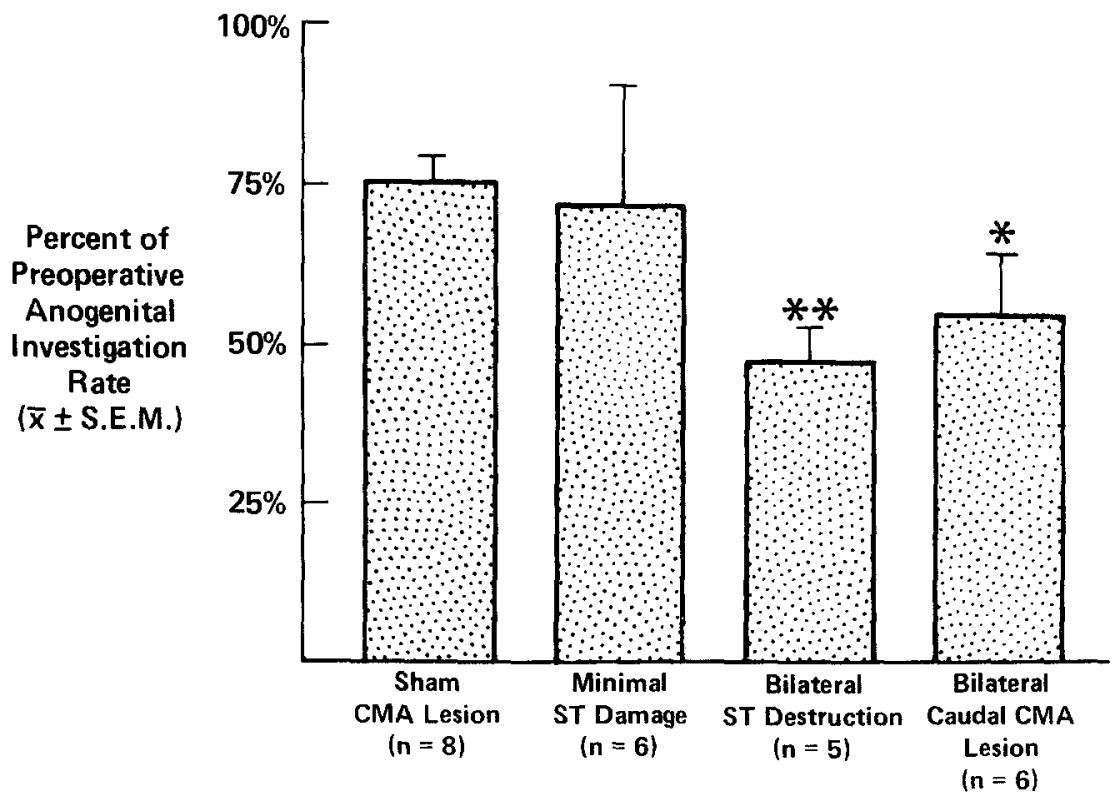

Fig. 6. Postoperative deficits in anogenital investigation displayed by male hamsters during mating tests, before and after sham lesions of the corticomedial amygdala (CMA), minimal damage to the stria terminalis (ST), bilateral lesions of the caudal CMA, or bilateral destruction of the ST. Percentage of preoperative rate of anogenital investigation was calculated by dividing the mean rate from all three postoperative tests, by the mean rate of both preoperative tests. ${ }^{* *} P<0.001$, ${ }^{*} P<0.05$, Mann-Whitney U-test compared to sham CMA lesion group. 
testing, since a decrease of similar magnitude in anogenital investigation rate may be seen in normal males over the course of successive mating tests (J.B. Powers, unpublished observation). Male hamsters with minimal ST damage also displayed a decrease in their postoperative rate of anogenital investigation, but that decrease was not significantly lower than that seen in animals with control lesions. In contrast, males with either bilateral ST destruction or caudal CMA lesions displayed a decrease of 53 and $45 \%$, respectively, in their postoperative rate of anogenital investigation, which was significantly lower than seen in males with sham CMA lesions. However, these deficits in anogenital investigation were not as severe as those previously observed in male hamsters with bilateral lesions of the medial amygdaloid nucleus. For example, in an earlier study [18] we reported that males with medial nucleus lesions showed a postoperative decrease of $85 \%$ in their rate of anogenital investigation.

Male hamsters with bilateral ST destruction also showed alterations during postoperative mating tests in their sniffing and licking investigation of other parts of the female's body. Males with bilateral ST destruction displayed a postoperative increase of over $100 \%$ in their investigation of the female's head and flank, from a mean rate of $2.6 \mathrm{sec}$ per test minute preoperatively to $4.5 \mathrm{sec}$ per test minute postoperatively $(P<0.001$, Wilcoxon $T$-test). Males with either sham lesions, caudal CMA lesions, or minimal ST damage showed no significant postoperative change in their rate of head and flank investigation of the female.

\section{DISCUSSION}

Although male hamsters with bilateral ST destruction, whether by electrolytic lesions or coronal knife cuts, displayed marked alterations in their temporal pattern of copulation, they did not show the complete absence of copulatory behavior or reduction in anogenital investigation displayed by males with bilateral lesions of the medial amygdaloid nucleus which were reported in previous studies [18, 19]. A majority of males with bilateral ST destruction continued to display copulatory behavior for up to two months following surgery, while in previous experiments, males with medial nucleus lesions stopped mating completely during all tests over a three month postoperative period. Furthermore, males with medial nucleus lesions showed a striking reduction in their postoperative rate of investigation of the female's anogenital region during mating tests [18, 19]. Male hamsters with bilateral ST destruction also displayed a decrease in their postoperative rate of anogenital investigation (Fig. 6), but this decrease was not as great in magnitude as that previously observed in males with medial nucleus lesions [18]. Caudal CMA lesions, which damaged the amygdaloid exit of the ST, led to alterations in copulatory and investigatory behavior similar to those after bilateral ST destruction, but less severe than those mating deficits seen after medial nucleus lesions. 
These results suggest that vomeronasal and olfactory information processed in the medial nucleus influences the display of male hamster mating behavior at least in part through non-strial efferents. Further studies in our laboratory have supported this hypothesis and indicate that some neurons of the medial nucleus project by way of a non-strial ventral pathway to the preoptic portion of the bed nucleus of the stria terminalis (BNST) [20], which, as previously mentioned, is another androgen-binding brain area implicated in the control of male copulatory behavior. Furthermore, recent behavioral experiments indicate that cutting these non-strial efferents, in combination with strial efferents of the medial nucleus, mimics the complete elimination of copulatory behavior and the investigatory deficits seen after medial nucleus lesions [21].

Even though bilateral destruction of the ST did not result in mating deficits equal to those seen after medial nucleus lesions, it is interesting to note that proportionately more male hamsters with bilateral lesions that damaged the medial ST stopped mating during a given postoperative test, than did males with complete bilateral destruction of the ST (see Fig. 1). This observation may be related to the topographic organization of amygdaloid efferents within the ST, as described from tract tracing studies using autoradiographic and silver degeneration techniques $[5,16]$. In the rat, the medial portion of the ST carries efferents from ventromedially-situated nuclei within the amygdala, primarily the medial nucleus, the anterior cortical nucleus, and the amygdalo-hippocampal area, while the lateral ST contains fibers from more dorsolaterally-placed nuclei, including the basolateral and central nuclei of the amygdala. Medial ST lesions in the hamster probably interrupted efferents from the medial nucleus, as well as the anterior cortical nucleus and amygdalo-hippocampal area, but may have left strial efferents from the basolateral and central nuclei intact. The more drastic effect of medial ST lesions may therefore be due to the presence of intact amygdaloid efferents from the basolateral and central nuclei in the lateral ST, which may have been responsible for a net inhibitory influence on the display of male copulatory behavior*.

Since the basolateral and central nuclei of the amygdala have been implicated in defensive and escape behavior in rodents and carnivores [13], it is possible that strial efferents from these areas might inhibit the male hamster's approach to the female and subsequent copulatory behavior because they convey complex sensory information about the female associated primarily with past aggressive encounters. It is not unlikely that certain stimulus characteristics of the female hamster may

\footnotetext{
* Caudal CMA lesions which damaged the amygdaloid exit of the ST led to copulatory deficits similar to those of male hamsters with medial ST lesions. Because of their medial location, caudal CMA lesions may have also left strial efferents from the laterally-placed central and basolateral nuclei intact, and in a similar manner their inhibitory influence may have led to a more frequent absence of copulatory behavior than that seen after complete ST lesions.
} 
be more closely tied to the male's defensive than sexual responses, since in this species the female is larger and dominant over the male, and, when sexually unreceptive, almost always displays intense aggressive behavior towards him [26]. From the male's perspective, during normal copulatory behavior, these aversive characteristics of the female are probably outweighed by the hedonic aspects of the female and her behavior associated with sexual activity, including her odor cues [3, 24] and ultrasonic vocalizations [10]. Vomeronasal and olfactory recipient areas of the amygdala, including the medial nucleus, may process odor cues that are hedonically associated with the female, since these same areas yield the highest rates of self-stimulation in the amygdala [33]. The net effect of medial ST lesions, then, might have been to inhibit the male's approach to the female and subsequent copulation, more often than complete ST lesions which interrupted both inhibitory and facilitatory sets of efferents.

When they did display copulatory behavior, male hamsters with both medial ST lesions and complete ST lesions showed alterations in their temporal pattern of copulation that are similar to those described in earlier studies of male rats with bilateral ST lesions [7, 11,30], and that may be attributed, at least in part, to a functional loss of vomeronasal and olfactory information transmitted by strial efferents of the medial nucleus and/or amygdalo-hippocampal area [19]. In normal animals, this chemosensory information probably facilitates not only that arousal which precedes copulatory behavior, but also the maintenance of a temporal pattern of intromissions leading to ejaculation. Male hamsters engage in vigorous sniffing and licking investigation of the female's anogenital region not only prior to the initiation of actual copulatory behavior, but also between intromissions and during the refractory period following ejaculation [2]. In addition, males are exposed to chemosensory stimuli from the female's vaginal secretion during the frequent grooming of their own anogenital region between intromissions [2].

Specific alterations in the copulatory behavior displayed by males with bilateral ST destruction may therefore be at least partly a consequence of the loss of relevant chemosensory cues during both appetitive (arousal) and consummatory (copulatory) phases of mating behavior. It is likely that cutting the ST also interrupts other types of amygdaloid-processed information relevant to the timing of copulatory behavior. The significant increase in mount latency displayed by these males during the first postoperative test may thus be a result of the loss of sexually-relevant chemosensory and other inputs which normally contribute to the male hamster's arousal preceding copulatory behavior. Similarly, the significant increase in the time between intromissions displayed by males with bilateral ST destruction may also represent a loss of relevant information, including odor cues, that normally facilitate the temporal pattern of intromissions leading to ejaculation. By the third postoperative test most of these males may have compensated for the loss of amygdaloid-processed information by relying more on tactile and other 
sensory stimuli provided by the female which may reach the BNST or MPOAH directly. Hence, mount latency returns to normal levels (Fig. 5A) perhaps because these male hamsters now rely on other sensory cues which contribute to their arousal preceding copulation, and the mean number of intromissions preceding ejaculation increases (Fig. 5C), perhaps because an increased amount of tactile stimulation is necessary to compensate for the loss of chemosensory and other inputs carried by the ST which normally facilitate the ejaculatory sequence.

It is interesting to speculate that a shift of emphasis in the nature of stimuli which contribute to that arousal preceding copulation, in males with bilateral ST destruction, may reflect synaptic changes in those neurons denervated of amygdaloid strial input which mediate the appetitive phase of mating behavior. Reinnervation of denervated post-synaptic sites by other axonal systems in close proximity to the denervated sites, has been demonstrated in the lateral septal nucleus of the adult rat [8] and it has been suggested that this phenomenon may occur in cells of the BNST denervated of strial input [9]. It is possible, therefore, that in animals with ST lesions, neurons deprived of strial input in the BNST and elsewhere are reinnervated with synaptic terminals from other afferents, and that these afferents may convey non-chemosensory information which modify the responsiveness of these cells. If the same neurons play a role in mediating the arousal preceding copulation, then synaptic changes making those neurons relatively more responsive to other female stimuli may provide a neural basis for the observed reduction in mount latency over the course of postoperative testing (Fig. 5A). These neural changes may account for at least one alteration in the temporal pattern of copulation displayed by male hamsters with bilateral ST destruction.

\section{ACKNOWLEDGEMENTS}

This research is supported in part by NRSA Grant T32-MH14279 to M.N.L. and by NIH Grants R01-NS14071 to S.S.W. and R0I-HD14535 to J.B.P.

\section{REFERENCES}

1 Adams, D.B., Brain mechanisms for offense, defense, and submission, Behav. Brain Sci., 2 (1979) 201-241.

2 Bunnell, B.N., Bolands, B.D. and Dewsbury, D.A., Copulatory behavior of golden hamsters (Mesocricetus auratus), Behaviour, 61 (1977) 180-206.

3 Darby, E.M., Devor, M. and Chorover, S., A presumptive sex pheromone in the hamster: Some behavioral effects, J. comp. Physiol. Psychol., 88 (1975) 496-502.

4 Davis, B.J., Macrides, F., Youngs, W.M., Schneider, S.P. and Rosene, D.L., Efferents and centrifugal afferents of the main and accessory olfactory bulbs in the hamster, Brain Res. Bull., 3 (1978) 59-72. 
5 De Olmos, J.S., The amygdala projection field in the rat as studied with the cupric silver method. In B.E. Eleftheriou (Ed.), The Neurobiology of the Amygdala, Plenum Press, New York, 1972, pp. 145-204.

6 Doherty, P.C. and Sheridan, P.J., Uptake and retention of androgen in neurons of the brain of the golden hamster, Brain Res., 219 (1981) 327-334.

7 Emery, D.E. and Sachs, B.D., Copulatory behavior in male rats with lesions in the bed nucleus of the stria terminalis, Physiol. Behav., 17 (1976) 803-806.

8 Field, P.M., Coldham, D.E. and Raisman, G., Synapse formation after injury in the adult rat brain: preferential reinnervation of denervated fimbrial sites by axons of the contralateral fimbria, Brain Res., 189 (1980) 103-113.

9 Field, P.M., Synapse formation after injury in the adult rat brain: failure of fimbrial axons to reinnervate the bed nucleus of the stria terminalis, Brain Res., 189 (1980) 91-101.

10 Floody, O.R., Behavioral and physiological analyses of ultrasound production by female hamsters (Mesocricetus auratus), Amer. Zool., 19 (1979) 443-455.

11 Giantonio, G.W., Lund, N.L. and Gerall, A.A., Effect of diencephalic and rhinencephalic lesions on the male rat's sexual behavior, J. comp. Physiol. Psychol., 73 (1970) 38-46.

12 Isaacson, R.L., The hypothalamus. In The Limbic System, Plenum Press, New York, 1974.

13 Kaada, B.R., Stimulation and regional ablation of the amygdaloid complex with reference to functional representations. In B.E. Eleftheriou (Ed.), The Neurobiology of the Amygdala, Plenum Press, New York, 1972, pp. 205-282.

14 Kevetter, G.A. and Winans, S.S., Connections of the corticomedial amygdala in the golden hamster. I. Efferents of the 'vomeronasal amygdala', J. comp. Neurol., 197 (1981) 81-98.

15 Kevetter, G.A. and Winans, S.S., Connections of the corticomedial amygdala in the golden hamster. II. Efferents of the 'olfactory amygdala', J. comp. Neurol., 197 (1981) 99-111.

16 Krettek, J.E. and Price, J.L., Amygdaloid projections to subcortical structures within the basal forebrain and brainstem in the rat and cat, $J$. comp. Neurol., 178 (1978) 225-254.

17 Larsson, K., Features of the neuroendocrine regulation of masculine sexual behavior. In C. Beyer (Ed.), Endocrine Control of Sexual Behavior, Raven Press, New York, 1979, pp. 77-163.

18 Lehman, M.N., Winans, S.S. and Powers, J.B., Medial nucleus of the amygdala mediates chemosensory control of male hamster sexual behavior, Science, 210 (1980) 557-560.

19 Lehman, M.N. and Winans, S.S., Vomeronasal and olfactory pathways to the amygdala controlling male hamster sexual behavior: autoradiographic and behavioral analyses, Brain Res., 240 (1982) 27-41.

20 Lehman, M.N. and Winans, S.S., Evidence for a ventral non-strial pathway from the amygdala to the bed nucleus of the stria terminalis in the male golden hamster (submitted).

21 Lehman, M.N. and Winans, S.S., Amygdaloid efferents to the bed nucleus of the stria terminalis and the medial preoptic area controlling male hamster sexual behavior: combined HRP and behavioral analyses, Soc. Neurosci. Abstr., 6 (1980) 460.

22 Malsbury, C.W. and Pfaff, D.S., Neural and hormonal determinants of mating behavior in adult male rats. A review. In L.V. DiCara (Ed.), Limbic and Autonomic Nervous System Research, Plenum Press, New York, 1974, pp. 85-136.

23 McBride, R.L. and Sutin, J., Amygdaloid and pontine projections to the ventromedial nucleus of the hypothalamus, $J$. comp. Neurol., 174 (1977) 377-396.

24 Murphy, M., Effects of female hamster vaginal discharge on the behavior of male hamsters, Behav. Biol., 9 (1973) 367-375.

25 Murphy, M. and Schneider, G.E., Olfactory bulb removal eliminates mating behavior in the male golden hamster, Science, 167 (1970) 302-303.

26 Payne, A.P. and Swanson, H.H., Agonistic behaviour between pairs of hamsters of the same and opposite sex in a neutral observation area, Behaviour, 36 (1970) 259-269. 
27 Sar, M. and Stumpf, W.E., Distribution of androgen-concentrating neurons in rat brain, In W.E. Stumpf and L.D. Grant (Eds.), Anatomical Neuroendocrinology, Karger, Basel, 1975 , pp. 120-133.

28 Scalia, F. and Winans, S.S., The differential projections of the olfactory bulb and accessory olfactory bulb in mammals, J. comp. Neurol., 161 (1975) 31-56.

29 Sheridan, P.J., The nucleus interstitialis striae terminalis and the nucleus amygdaloideus medialis: prime targets for androgen in the rat forebrain, Endocrinology, 101 (1979) 130-136.

30 Valcourt, R.J. and Sachs, B.D., Penile reflexes and copulatory behavior in male rats following lesions in the bed nucleus of the stria terminalis, Brain Res. Bull., 4 (1979) 131-133.

31 Velasco, M.E. and Taleisnik, S., Effects of the interruption of amygdaloid and hippocampal afferents to the medial hypothalamus on gonadotropin release, J. Endocrinol, 51 (1971) 41-55.

32 Winans, S.S. and Powers, J.B., Olfactory and vomeronasal deafferentation of male hamsters: histological and behavioral analyses, Brain Res., 126 (1977) 325-344.

33 Wurtz, R.H. and Olds, J., Amygdaloid stimulation and operant reinforcement in the rat, $J$. comp. Physiol. Psychol., 56 (1963) 941-949. 